\title{
L'expertise internationale en Afrique : le cas de l'expertise juridique sur les questions foncières
}

\section{Etienne Le Roy}

\section{(2) OpenEdition}

\section{Journals}

Édition électronique

URL : http://journals.openedition.org/apad/344

DOI : 10.4000/apad.344

ISSN : 1950-6929

Éditeur

LIT Verlag

Édition imprimée

Date de publication : 15 décembre 1991

\section{Référence électronique}

Etienne Le Roy, «L'expertise internationale en Afrique : le cas de l'expertise juridique sur les questions foncières », Bulletin de l'APAD [En ligne], 2 | 1991, mis en ligne le 28 juin 2006, consulté le 08 septembre 2020. URL : http://journals.openedition.org/apad/344 ; DOI : https://doi.org/10.4000/apad.344

Ce document a été généré automatiquement le 8 septembre 2020

Bulletin de I'APAD 


\title{
L'expertise internationale en Afrique : le cas de l'expertise juridique sur les questions foncières
}

\author{
Etienne Le Roy
}

1 Je voudrais soutenir ici que ce qui légitime l'intervention de l'assistance technique au titre d'une expertise est un mélange de connaissances techniques, d'efficacité bureaucratique, de cécité politique ou d'irresponsabilité éthique qui n'est pas un donné mais un acquis culturel complexe qui doit faire une très large place à l'idéologie.

2 C'est ainsi la leçon d'une expérience personnelle de ces cinq dernières années que je souhaite tirer en montrant, d'une part, qu'on ne peut devenir expert international qu'en raison de la capacité de traduire un savoir technique dans un savoir-faire politique. L'habilité de l'expert tient aussi à l'art de savoir prendre les situations et les dossiers "dans le sens du poil", c'est-à-dire selon les attentes implicites des commanditaires, afin que soient justifiées les décisions qu'ils seront amenés à prendre et à imposer. Mais, d'autre part, on ne peut se maintenir expert qu'en contrôlant la concurrence ou en régulant l'intervention d'autres collègues.

3 Peu organisée et mal identifiée, la profession des experts internationaux est en fait, discrètement, organisée selon les lois du marché. Si l'offre dépasse souvent la demande (et les capacités de financement), il y a des situations où, inversement, les spécialistes sont peu nombreux et peuvent ainsi imposer leurs conditions en particulier pour la réalisation et l'utilisation des études qui leur sont commandées.

4 La possibilité, par le marché de l'expertise, de faire émerger des problématiques nouvelles (ou, au contraire, d'interdire certaines pratiques) est le second aspect que je voudrais illustrer à partir de mon expérience.

5 Je voudrais ainsi, en conclusion, retenir l'idée que l'expert est, dans le contexte de l'assistance technique aux pays en développement, dans la position d'une éminence grise, c'est-à-dire d'un pouvoir qui n'ose pas dire son nom. Serait-ce le signe du maintien ou d'une renaissance d'un rapport de domination coloniale?

Expert juriste : entre le commentaire doctrinal et la tentation du divin législateur 
6 Parler de ces situations et dessiner brièvement une biographie de l'expert à l'état naissant, c'est, bien entendu, prendre certains risques. Sans mettre en question une "loi du silence" qui suggérerait "l'omerta", c'est-à-dire une analogie directe avec une "maffia" ou un "milieu", il y a au moins un devoir de réserve qui est attendu de l'expert international et qui est souvent explicitement imposé par des clauses contractuelles lors de son recrutement. L'étude ou le rapport est la propriété de l'organisation internationale, du bureau d'études ou de l'État commanditaire et ne peut être cité que sous les réserves d'usage. Ceci souligne qu'on ne peut mettre en cause des institutions et des systèmes de décision qu'en acceptant la sanction possible du dévoilement de ces "enjeux d'institutions" (pour reprendre une expression forte de Pierre Legendre): l'exclusion.

7 Ayant ainsi dégagé les bornes que je ne saurais dépasser, sauf à être "hors jeu" (ce qui est plus facile à accepter quand l'expertise n'est qu'un métier second par rapport à une profession principale), je vais illustrer ici. à partir de mon expérience d'expert-juriste, les relations existant entre mon savoir de juriste et le pouvoir du politique, et comment celui-ci a cherché à utiliser certains aspects de mon savoir pour ses propres fins, l'assistance à la décision, tout en devant accepter, dans certaines circonstances, une délégation de son propre pouvoir à l'expert.

Le besoin d'expertise pour l'assistance à la décision.

"Être ou ne pas être expert, là est la question". C'est au moins rétrospectivement la réflexion qu'on peut faire sur les conditions en fonction desquelles on passe du statut de chercheur à celui d'expert et d'une recherche universitaire fondamentale à ses applications.

9 On s'aperçoit en effet, dans les situations que j'ai eues à expérimenter, que c'est moins la qualité des savoirs que leur nature, utilitaire, qui a été le critère déterminant de la prise en considération d'une habilité particulière et ainsi de mon utilisation en qualité d'expert. Spécialiste des questions foncières en Afrique noire, $\mathrm{j}$ 'avais développé durant une quinzaine d'années des recherches sur le "droit foncier coutumier" qui paraissaient non seulement originales mais surtout essentielles pour l'élaboration de politiques législatives soucieuses d'une meilleure prise en compte des réalités sociologiques et des modalités "endogènes" de régulation des rapports sociaux. Ces analyses, auxquelles j'avais consacré deux thèses, de multiples travaux en séminaires et de nombreuses publications, non seulement n'intéressaient personne mais également m'avaient valu quelques ennuis, ainsi au Sénégal entre 1970 et 1975 où j'avais été écarté des recherches de terrain.

10 Tout en approfondissant et en élargissant ces recherches, il devenait évident, à la fin des années soixante-dix, que, non seulement je n'avais pas d'espoir que leurs résultats puissent être pris en considération, mais qu'en outre elles étaient "gênantes" parce qu'elles remettaient en cause les politiques foncières préconisées au nom du développement. Celles-ci, reposant sur deux dogmes, le monopole foncier de l'Etat et/ ou la nécessaire diffusion de la propriété privée civiliste, ne pouvaient être légitimées et concrétisées qu'à condition que le droit foncier coutumier soit, de son côté, délégitimé et, explicitement ou non, effacé au profit du nouveau Droit.

11 Ce travail de réinterprètation et de relégitimation avait débuté dès la période coloniale pour aboutir à une présentation caricaturale que la préparation de "journées d'études sur les problèmes fonciers en Afrique" en 1980, avait permis de caractériser sous l'expression "le référent précolonial", comme image dévalorisante des rapports 
fonciers traditionnels ${ }^{1}$. L'enjeu politique était en effet, et reste souvent, de concrétiser le rôle de l'État et l'effacement des conceptions millénaires au nom des exigences du développement, de l'intégration nationale, etc.

Ainsi, ce n'est que lorsque le réseau international de chercheurs, fondé à la suite des journées d'études de 1980, s'est préoccupé des politiques étatiques, dans le cadre d'un rapport commandité par le ministère de la coopération en $19822^{2}$ que notre recherche fondamentale a commencé à intéresser des organismes internationaux. De demandes d'informations en propositions plus explicites et alléchantes, notre réseau de chercheurs a ainsi été testé et j'ai été sollicité pour participer à un colloque international en qualité de rapporteur général. La réussite, apparente, de ce colloque, a conduit à solliciter mon intervention dans d'autres colloques en qualité de consultant, puis à me confier une mission d'évaluation des politiques foncières où mon statut d'expert-juriste était reconnu. Enfin, et parallèlement au déroulement de ce travail, j'ai été envoyé aux Comores pour faire des propositions de politique foncière dans le cadre d'un programme de coopération technique. Il y a là un enchaînement de situations qui a provoqué graduellement une transformation de ma fonction de chercheur universitaire en expert international, avec des limites implicites dont je me suis rendu compte au fur et à mesure de la communication des résultats à l'organisation qui m'employait. Mon domaine d'intervention était en effet vaste dans la mesure où non seulement il n'y avait pas de travaux généraux mais où, également, la documentation était à réunir, les textes juridiques étant le plus souvent mal publiés et inconnus à l'échelle internationale. Il fallait à la fois collationner les textes, en proposer une interprétation, en évaluer les incidences et en mesurer le degré d'application. L'organisation attendait de moi une compilation de ces textes et une démarche de commentateur sans aller jusqu'au travail de la doctrine juridique, c'est-à-dire sans faire de propositions de politique juridique. Au nom d'une "neutralité" - qui n'est que la traduction de la réalité d'une organisation fonctionnant comme un club d'États soucieux les uns vis-à-vis des autres de non-ingérence dans les affaires d'autrui - on attendait de mon travail qu'il éclaire des choix de politiques déjà mises en œuvre sans expliquer pourquoi ces politiques avaient très généralement échoué.

13 Au-delà des difficiles négociations avec certains membres de cette organisation pour faire publier l'essentiel de mes résultats, ce qu'on retiendra ici c'est que l'expertise sollicitée n'avait pas pour objectif de répondre à l'idée - naïve - que j'en avais : "savoir c'est pouvoir", mais avait une utilité plus "bureaucratique", permettant de justifier à l'égard des États membres l'intérêt que prenait l'organisation à certaines questions sensibles tout en garantissant aux États occidentaux principaux bailleurs de fonds que les solutions préconisées par ladite Organisation ne remettaient pas en cause les politiques de coopération bi ou multilatérales. Dans la logique des services de l'organisation, pris entre les besoins d'intervention sur le terrain et les exigences budgétaires, certaines expertises devaient d'abord justifier leur existence avant de résoudre des problèmes de fond.

14 A mon corps défendant, je me suis ainsi aperçu que l'expert n'était pas sollicité pour transposer son savoir (recherche fondamentale) en savoir-faire (recherche juridique appliquée) mais pour légitimer par son statut et sa science la (re)production de rapports de pouvoir. C'est finalement à ma méconnaissance de la nature réelle des relations internationales que je me heurtais puisque les critères de l'organisation sont conformes à sa capacité d'intervention, donc "logiques" dans sa perspective. 

l'expertise, ne doit pas être généralisée. Car des interventions ultérieures dans le cadre d'autres organismes m'ont permis de réinterpréter ces difficultés comme particulièrement liées à la crise financière que connaissait cette organisation. En effet, si les logiques institutionnelles interfèrent toujours avec la prise en considération de l'expert et de ses résultats, plus substantiellement ce sont des logiques décisionnelles qui l'emportent parce qu'on attend de l'expert que, tout en renforçant la position de l'institution qui l'emploie, on puisse justifier les décisions qu'on sera amené à prendre, de faire ou de ne pas faire, en mobilisant la science au service du politique. confusion des rôles peut apparaitre, le savoir se transformant alors en pouvoir.

Le pouvoir du savoir : l'expert juriste comme divin législateur.

17 J'emprunte à nouveau à Pierre Legendre l'expression du "divin législateur" par laquelle il traduit cette dimension sacrale attachée à la fonction législative dans la tradition occidentale moderne. Dans la mesure où notre modèle de l'Etat a été conçu sur le modèle de Dieu élaboré à l'occasion de la Contre-Réforme lors du Concile de Trente, la production de normes juridiques transpose dans le contexte laïque les représentations de la transmission biblique du Décalogue. Le législateur est impliqué dans ce processus de codification que $P$. Bourdieu dénomme "mettre en forme et mettre des formes" 3 , au nom d'un principe d'autorité qui a été dénommé Dieu, la Raison puis l'Intérêt général, mais qui a toujours un lien avec le sacré. Cette dimension sacrale de la fonction législative reste parfaitement abstraite et apparemment anecdotique tant qu'on n'a pas supporté soi-même et de façon solitaire, le poids de la responsabilité qu'implique la conception et la rédaction de nouveaux principes juridiques. présenter les axes, mais de réfléchir à la responsabilité de l'expert qui, en raison des circonstances, non seulement tient la plume du politique - ce que font les rédacteurs de l'Assemblée nationale et du Sénat en France - mais risque aussi de voir les textes rédigés adoptés tels quels par l'organe délibérant en raison d'une perfection technique supposée mais que l'auteur est amené le premier à reconsidérer.

19 A la suite de la mission initiale d'expertise foncière aux Comores où j'avais eu à évaluer les besoins et à proposer, sous forme de scénarios, les diverses politiques qui s'offraient à la République fédérale islamique des Comores, il m'a été demandé de tester l'applicabilité de deux des sept scénarios retenus par le chef de l'Etat en fonction de leur conformité avec les objectifs économiques et avec l'évolution constatée des mentalités et des comportements. Assisté d'un bureau d'études, je n'ai eu aucune peine à démontrer que l'une de ces options dépassait très largement les moyens financiers de l'Etat comorien. Aussi m'a-t-il été demandé, lors d'une nouvelle mission, de concrétiser les principes orientant le seul scénario opérationnel.

Cependant, dans le contexte spécifique de la RFIC, venue tard à l'indépendance et souffrant cruellement d'un très grave retard tant dans la formation des hommes que dans la réalisation des infrastructures du développement, il n'y avait pas localement de juriste assez expérimenté dans la rédaction du Droit nouveau et il a donc fallu que l'expert, devenu conseiller du prince, passe de la désignation des objectifs à la concrétisation des normes. Ce faisant, j'avais l'occasion, inimaginable auparavant, d'appliquer concrètement les principes de politique juridique que j'avais élaborés 
antérieurement, au risque de transformer mon écriture juridique en une expérience de laboratoire.

21 Le choix, là, n'était plus technique ou scientifique mais bien politique, et c'est bien ainsi que j'abordais la mission qui m'était confiée. Si j'y ai répondu affirmativement par des choix qui privilégiaient une loi d'orientation et des textes d'accompagnement plutôt qu'un code foncier, si j'ai élaboré le concept de patrimoine foncier national pour régler le statut des terres non domaniales ni immatriculées, si j'ai refusé de privilégier, sans en interdire le développement, la propriété civiliste, si, enfin, j'ai cherché à associer la pensée juridique islamique de rite chafi'iste à la philosophie juridique, c'est chaque fois en fonction de considérations idéologiques (personnelles) et politiques (à l'échelle de la RFCI et de ses relations internationales). Tout en maîtrisant, sans doute mieux que d'autres, les connaissances du droit coutumier, du droit colonial, de la sharia et des expériences réformatrices de l'Afrique continentale, ce n'est pas en "savant" mais en politique ${ }^{4}$ que j'ai réglé le dispositif juridique. Car, en faisant des choix et en sachant que le personnel administratif serait dépassé par la complexité des problèmes qu'il fallait conjointement prendre en considération pour arriver à des solutions simples et pragmatiques, j'imposais le pouvoir de mon savoir, au risque de l'erreur.

Si une telle erreur n'est jamais définitive et qu'il est possible aux hommes de défaire ce que l'un d'eux à fait, on comprendra que, en conscience, l'expert interpellé par la solitude du décideur ait passé quelques nuits blanches à vérifier ses montages et sa machinerie, comme l'ingénieur en chef de l'Aérospatiale responsable de l'envoi d'une fusée Ariane dans l'espace. Ajoutons enfin que cette solitude, pesante sur le plan de la prise de décision, était la contrepartie du monopole dont je disposais à l'échelle de l'expertise internationale, seul à m'être exposé au risque de traiter au fond le problème foncier de la RFCI, considéré comme "impossible" à régler par mes confrères.

Je retirai ainsi les avantages, intellectuels, d'une situation de monopole sur le marché de l'expertise.

Le pouvoir du marché, l'argent des autres et l'éthique professionnelle.

24 Les expériences que j'ai évoquées tendraient à souligner que si le savoir de l'expert est toujours invoqué, sans être réellement utilisé parfois, ce n'est pas en raison de la qualité du chercheur, de la profondeur des travaux ou de l'intelligence des solutions qu'on recrute un expert. Ce qu'on recherche c'est un savoir-faire qui peut avoir pour objectif soit de confirmer les intentions de décision du politique ou du bailleur de fonds (qui fait également de la politique), soit d'en modifier des termes, voire exceptionnellement se substituer en partie à lui.

La première situation, étant la plus commode, est particulièrement recherchée mais doit être concrétisée par la possibilité d'obliger l'expert à accepter non seulement les termes de référence mais aussi les intentions plus ou moins explicites qui les accompagnent et qu'un expert senior est en mesure d'identifier au cours de l'entretien de mise en route ${ }^{5}$ mais que le junior ne découvre parfois que trop tardivement, lors de la séance de "debriefing" ${ }^{6}$. Pour ce faire, il faut que la concurrence la plus large règne dans l'offre d'expertise, de telle manière que les organismes demandeurs et financeurs puissent sélectionner les candidats les plus dociles (et les plus sensibles à la menace de non recrutement) pour imposer, même inconsciemment, les conclusions conformes à leurs attentes. déséquilibré où l'offre d'expertise peut être d'autant plus manipulée que la profession 
n'est pas régulée (les institutions peuvent ainsi toujours susciter de nouvelles vocations et constituer le vivier de leurs experts). Par ailleurs, le marché s'ouvre, logiquement, aux chercheurs-experts du tiers monde, parfois, comme ce fut le cas à l'UNESCO, de manière intentionnelle, pour corriger des excès de favoritisme antérieur. L'offre est donc en croissance exponentielle puisqu'il y a toujours plus de diplômés et aussi peu de critères explicites de sélection.

Parallèlement, la demande d'expertise se rigidifie ou diminue pour plusieurs raisons. Tantôt on préfère recourir à des évaluations internes plutôt qu'internationales parce que plus discrètes et moins coûteuses. Les États comme les organismes internationaux utilisent leur personnel aux lieu et place d'un consultant externe. Tantôt l'expertise est une condition liée de l'allocation d'aide ou de financement de projet. Dans ce cas, le plus souvent, le bailleur de fonds impose son expert national et dans certains organismes on partage le "gâteau" des expertises à la hauteur des apports financiers de chacun des états donateurs. Tantôt, enfin, et surtout durant les années 1980-1985, les budgets des institutions du système des Nations-Unies ont connu des contractions sévères sous l'effet de l'application des doctrines néo-libérales et du désengagement politique des U.S.A. et de la Grande-Bretagne $d$ "'arènes" trop idéologiques comme l'UNESCO. La dévaluation du dollar, monnaie de référence du système des Nations Unies, la décroissance des budgets et l'envol des frais de mission impliquaient une raréfaction de nombre d'expertises et certaines modifications: durée raccourcie, condition de dépôt des conclusions plus draconiennes, frais mieux contrôlés, obligation fréquente de réaliser, au cours d'une même mission, plusieurs opérations peu conciliables (relevant parfois de directions différentes de l'organisme) mais imposées par les conditions de financement de l'expertise.

Le déséquilibre d'un tel marché entraîne certaines conséquences pour les experts. Nous allons voir quelles réponses ils apportent de manière plutôt individualiste. Puis nous évoquerons une situation, exceptionnelle mais favorable aux experts, quand une demande enfle dans un domaine et qu'un petit nombre d'experts sont en situation d'y répondre. Dans ce cas, un minimum de cohésion peut assurer un monopole d'expertise qui peut déboucher sur deux objectifs : un drainage sélectif de "l'argent des autres" en raison de la puissance du réseau ainsi créé, ou la valorisation par ce monopole d'une éthique professionnelle exigeant des conditions plus qualitatives et des applications plus crédibles. Bien que ces deux options ne soient pas totalement contradictoires, il faut choisir, et je dirai quelques mots des conditions dans lesquelles se développe une déontologie dans le contexte des recherches et études foncières.

Les experts face au pouvoir de l'argent

Les enjeux financiers poussent à une spécialisation.

L'expert indépendant et les autres. L'expert-type, à mes yeux, est celui que la profession dénomme un "free lance", collaborateur indépendant vivant entièrement de ses expertises et relevant en France des professions libérales Notons, ce n'est pas tout à fait anecdotique, que le terme désignait initialement les gardes suisses propriétaires de leur hallebarde et se vendant aux princes allemands tantôt catholiques, tantôt protestants. Ce que nous dénommons des mercenaires.

31 Selon une opinion dominante, trois mois de missions d'expertise suivis contractuellement de trois mois de rédaction de rapports suffisent à assurer des revenus annuels satisfaisants, si on n'a pas de charge de famille. Ce type d'expertise est en effet plutôt masculin, réservé à des seniors, célibataires ou dont le conjoint est 
salarié et autonome. Dans certains organismes, il est même typiquement "wasp" (white-anglo saxon-protestant). Il faut en effet une grande disponibilité (matérielle et intellectuelle), une opiniâtreté dans la démarche et s'adapter à la très rude concurrence professionnelle, à l'américaine, où le plus fort l'emporte parce que le plus fort.

Les autres experts sont tantôt des anciens qui ont charge d'âmes ou sont lassés de voyager perpétuellement aux quatre coins du monde dans des hôtels également anonymes. Tantôt ce sont des nouveaux venus qui ont une profession principale et recourent à l'expertise comme un deuxième métier (voire un troisième, dans certains pays du tiers-monde). Pour certains, enfin, c'est un de ces beaux-arts qui permettent de voir le monde ou d'affronter des interlocuteurs nouveaux. De jeunes retraités suivent fréquemment cet itinéraire. Ces esthètes de l'expertise n'en sont pas moins aussi efficaces et parfois plus libres de pressions bureaucratiques.

L'expert généraliste et l'expert spécialiste. Comme en médecine, le spécialiste remplace progressivement le généraliste sous l'effet de la concurrence. Il y a eu un temps où le fait d'avoir occupé une fonction administrative donnait compétence à être expert du transfert de techniques de gestion dans le domaine considéré. On ne se préoccupait pas de savoir si le contexte pouvait accepter le transfert et si l'habilité de l'expert était suffisante pour que les résultats soient appropriables. La concurrence et les exigences nouvelles ont introduit plusieurs critères de spécialisation, par régions, par disciplines et par thèmes.

La spécialisation par région est la plus ancienne, coïncidant en partie avec des regroupements de scientifiques en africanistes, américanistes, etc. Sous l'effet de la concurrence les distinctions se sont affinées, par divisions sous-continentales, voire étatiques, par exemple pour le Mozambique ou l'Angola lorsque les conditions récentes ont limité à quelques spécialistes l'accès au terrain. Dans nombre de cas, des critères linguistiques s'ajoutent à ces distinctions géographiques. Parler swahili, peul ou lingala peut être le facteur de sélection le plus décisif.

La caractérisation par disciplines s'est cependant le plus souvent répandue dans la mesure où les conventions universitaires de répartition des savoirs et des apprentissages sont devenues un critère généralisé de reconnaissance sociale. Le choix de l'expert selon sa discipline de formation et (ou) de pratiques professionnelles est d'une grande efficacité lorsque l'objet de l'expertise est monodisciplinaire ou qu'il peut être subdivisé en autant d'expertises que de disciplines impliquées.

Lorsque l'objet de l'expertise est pluri ou interdisciplinaire, il faut recourir à des experts spécialisés sur ce thème. Prenons l'exemple du foncier, particulièrement illustratif d'une spécialisation par thème. Le foncier est un rapport social purement imaginé et variable de société en société à partir des représentations qui lui sont propres du monde et des projets que les hommes y développent. Ce rapport social est construit, dans toutes les sociétés où nous avons travaillé, à partir de trois facteurs pondérés par un quatrième: du juridique plus de l'économique, plus des techniques d'aménagement, le tout pondéré par du politique. Aucune discipline ne peut donc prétendre monopoliser un tel champ d'étude et, lorsque les disciplines à maîtriser sont substantiellement diverses, l'expertise ne peut plus être individuelle mais collective. Une expérience récente au Mali montre en effet que la seule façon d'aborder dynamiquement la question foncière est, d'une part, de réunir spécialistes du Droit, de l'Économie et, dans ce cas, de l'hydraulique pastorale et, d'autre part, de mélanger 
experts nationaux et experts étrangers pour tenir compte de toutes les facel1es des facteurs politiques nationaux et internationaux.

Le facteur "rémunération" est un critère essentiel pour comprendre non seulement la dépendance de l'expert à l'égard de son financier, mais également le type de produit, le rapport d'expert, lequel est une marchandise.

Le type de mission dont est chargé l'expert, le montant des sommes engagées (selon la possibilité de jouer sur le marché et d'imposer des taux, des indemnités ou des frais de séjour qui peuvent permettre de vivre confortablement) font que le rapport professionnel n'est pas identifiable à un salariat banal. Les contrats sont à durée très déterminée et de multiples garanties ${ }^{7}$ sont prises pour éviter que l'expert ne se considère comme appartenant au personnel de l'agence. La relation professionnel le est plus souvent celle d'un artiste (privé le plus souvent d'un impresario) avec un directeur de troupe ou de théâtre. Il faut plaire, non par son physique avantageux ou l'art de déclamer, mais par le contenu du rapport. C'est le rapport qui est acheté, afin d'être revendu sur d'autres marchés à forte teneur symbolique ainsi qu'on a pu le montrer dans le début de ce texte (au sein de l'institution ou dans les conflits inter-institutionnels).

Ainsi, non seulement le rapport d'expertise cesse d'être un document scientifique pour devenir une marchandise avec ses standards propres, mais peut également avoir certaines fonctions d'une monnaie à usage spécialisé. De même qu'autrefois, dans l'Afrique précoloniale, les bandes de cotonnades ou les barres de fer ne servaient qu'à certains types d'échanges (matrimonial par exemple) au sein de réseaux spécialisés (dits réseaux de prestige), de même peut-il en être ainsi pour les rapports d'expertise. Ils ne circulent qu'entre certains acteurs, ne sont conçus que selon des termes de référence stéréotypés et n'ont pas pour fonction de changer le système de décision mais d'échanger (le plus souvent des messages techniques, parfois d'autres informations).

40 Ces considérations paraitront sans doute pessimistes, mais correspondent à une situation objective et à des rapports de force qu'on ne doit pas mésestimer.

Organiser le marché selon une éthique professionnelle.

41 Organiser le marché peut être ressenti par les recruteurs comme par les candidats-experts lorsqu'un produit nouveau est susceptible de réorienter certaines parts du marché. C'est ce qui arrive actuellement avec les études foncières. Longuement négligées, elles apparaissent maintenant clairement comme une des conditions des futurs projets de développement. Ainsi, le club du Sahel a introduit ces études parmi les trois priorités fixées lors du séminaire de Segou (Mali) en 1989 pour les années à venir, avec l'écologie et la décentralisation.

Pour éviter un dérapage dans la réalisation de ces études, un projet de "consortium" a ainsi été conçu, à la double initiative d'employeurs et d'employés potentiels, relevant du secteur public, privé ou associatif. Pour imposer un critère de qualité (en particulier interdisciplinaire) et éviter que l'expertise ne devienne qu'une marchandise sans usage dans des processus endogènes de développement, diverses initiatives sont en cours d'approfondissement, dont on n'anticipera pas ici la démonstration pour ne souligner qu'une idée banale ailleurs, peut-être décisive ici. L'idéal syndical permettrait en effet de rompre le caractère segmenté et individualiste du marché pour non seulement défendre les intérêts matériels des experts, mais également leur faire prendre 
conscience des enjeux économiques et politiques d'une meilleure efficacité de leurs interventions. ${ }^{8}$

C'est, en tout cas, sur ces questions, que je souhaite conclure. Cette réflexion sur l'expertise à l'échelle internationale dans le contexte historique très particulier de la décennie quatre-vingt, marquée en Afrique par la crise des économies et des États, a balancé entre deux visions, nullement contradictoires.

Dans une perspective réaliste, qui peut facilement tourner au cynisme, l'expertise objective un rapport de force d'une part entre les États du Tiers Monde et les pays développés, via les organismes internationaux, et, d'autre part, l'offre et la demande de savoir-faire, de savoir pour décider. Dans ce contexte, l'expert est un mercenaire de luxe dont l'intervention est structuralement liée à une fonction de communication en codant l'échange des messages entre les instances internationales de financement et les administrations nationales.

Dans une perspective plus optimiste qui ne doit pas devenir un idéalisme béat, l'expert, tout en reconnaissant les déterminismes économiques et politiques pesant sur son activité, peut en jouer individuellement et collectivement. Nous avons vu que des réponses collectives sont encore rares et ne pourraient sans doute concerner qu'une part des expertises, là où "technique" et "politique" peuvent être mesurées à l'aune d'une éthique professionnelle.

Ces deux approches sont sans doute originales par rapport à l'image que l'on a souvent de l'expert judiciaire, notabilité attachée à un tribunal et dont l'intervention est réputée tant pour sa qualité que pour sa rentabilité. Or cette neutralité, qui n'est sans doute qu'apparence dans l'expertise judiciaire française, vole en éclat dans le contexte des organisations internationales, moins en raison d'une perversité singulière de ces organismes qu'en raison du rôle qu'ils assument dans les relations inter-étatiques et des logiques bureaucratiques qui justifient et déterminent leurs interventions.

Remplissant une fonction d'articulations entre les besoins exprimés à l'échelle nationale et les régulations qui émergent à l'échelle essentiellement mondiale (les institutions monétaires internationales issues du système de Bretton Wood en particulier), ils assument la double obligation de traduire et de transmettre les messages dans les deux sens. Plus ces messages sont délicats ou dangereux, plus ils devront être codifiés et plus le rôle de certains experts (au moins en diplomatie) s'avèrera décisif. Pour utile et même indispensable que soit ce système international de communication par experts interposés, il n'en comporte pas moins des limites. Je n'en évoquerai que trois pour conclure: 1) C'est un système peu démocratique dans la mesure où les décisions fondées sur les "dires" de l'expert sont peu, mal ou pas du tout soumises au verdict des citoyens. 2) C'est un système qui valorise les niveaux de décision à l'échelle internationale en sous-évaluant, voire en ignorant, les déterminations à l'échelle locale.(on a pu montrer ailleurs ${ }^{9}$ que c'est l'absence de complémentarité entre ces systèmes de décision et leurs logiques contradictoires qui expliquent l'échec de processus de développement) 3) Enfin, et sans remettre en question les besoins de transfert de connaissances exogènes et de bilans critiques extérieurs des politiques de développement, l'usage actuel des expertises paraît trop lié à une vision au mieux paternaliste, au pire néo-coloniale des rapports entre les pays du sud et les pays du nord. 
C'est là, sans doute, où il ne conviendrait pas que le cynisme s'installe et que l'Afropessimisme l'emporte sur le marché des valeurs de civilisation.

\section{NOTES}

1.Voir E. Le Bris, E. Le Roy, F. Leimdorfer. Enjeux fonciers en Afrique noire, Paris, ORSTOM-Karthala, 1982, p.23-26.

2.E. Grégoire, E. Le Bris, E. Le Roy. Politiques foncières étatiques en Afrique francophone. Enjeux et perspectives. Paris, Rapport au ministère de la coopération et du développement, 1982, 280p.

3.P. Bourdieu. "Habitus, code et codification". Actes de la recherche en sciences sociales. Sept.1986, vol. 64, p.41.

4.Max Weber. Le savant et la politique. Paris, UEG col. 10-18, trad. fse, 1963, 159 p. Dans le contexte comorien, je restais ainsi un expert aux yeux de l'organisme qui me rémunérait et qui gardait le pouvoir d'approuver ou non mon travail, et considéré comme le "monsieur-foncier-du-Président" pour le personnel administratif.

5.La connaissance de ces attentes implicites, des rapports d'expertise précédents et des données collectées au siège permet ainsi à l'expert de réduire considérablement ses problématiques et d'anticiper rapidement certaines conclusions. Une des histoires (jokes) du métier raconte comment un expert avait réussi à programmer son micro-ordinateur de telle façon que l'essentiel du texte du rapport était composé avant l'arrivée sur le terrain où le travail consistait à compléter avec des statistiques locales les quelques "blancs" du texte. Comme toute blague, cette histoire renseigne d'abord sur ceux qui la racontent.

6.Le franglais est ici de rigueur comme une langue initiatique, surtout entre experts français.

7.Aussi l'expert doit assurer sa propre couverture sociale. Pour l'expert "free lance" victime d'un accident de travail, la question est réellement préoccupante.

8.Cette auto-organisation de la profession est réalisée, en dehors des associations à but très spécialisé ou des syndicats de bureaux d'études (bien discrets en temps de crise), par l'AITEC regroupant dans une association unique ingénieurs, techniciens et experts en matière de coopération (14, rue de Rungis, 75014 Paris).

9.E. Le Roy, "Oublier Babel. Une approche interculturelle des politiques de développement rural à travers l'exemple de la seconde génération de réformes agro-foncières en Afrique francophone", Bruxelles, Fondation ACP/CEE pour la coopération culturelle, Colloque Culture et Agriculture, Amsterdam, déc. 1987, à paraître. 


\section{AUTEUR}

\section{ETIENNE LE ROY}

Université Paris I-APREF A 